Louisiana State University

LSU Digital Commons

Faculty Publications

Department of Physics \& Astronomy

$1-1-1997$

\title{
Generation of attosecond pulses in macroscopic media
}

\author{
Philippe Antoine \\ Université Catholique de Louvain \\ Dejan B. Milošević \\ Université Catholique de Louvain \\ Anne L'Huillier \\ Lunds Tekniska Högskola \\ Mette B. Gaarde \\ Lunds Tekniska Högskola \\ Pascal Salières \\ CEA Saclay
}

See next page for additional authors

Follow this and additional works at: https://digitalcommons.Isu.edu/physics_astronomy_pubs

\section{Recommended Citation}

Antoine, P., Milošević, D., L'Huillier, A., Gaarde, M., Salières, P., \& Lewenstein, M. (1997). Generation of attosecond pulses in macroscopic media. Physical Review A - Atomic, Molecular, and Optical Physics, 56 (6), 4960-4969. https://doi.org/10.1103/PhysRevA.56.4960

This Article is brought to you for free and open access by the Department of Physics \& Astronomy at LSU Digital Commons. It has been accepted for inclusion in Faculty Publications by an authorized administrator of LSU Digital Commons. For more information, please contact ir@lsu.edu. 


\section{Authors}

Philippe Antoine, Dejan B. Milošević, Anne L'Huillier, Mette B. Gaarde, Pascal Salières, and Maciej Lewenstein 


\title{
(19:3) \\ LUND UNIVERSITY
}

\section{Generation of attosecond pulses in macroscopic media}

\author{
Antoine, P; Milosevic, D. B; L'Huillier, Anne; Gaarde, M. B; Salieres, P; Lewenstein, M \\ Published in: \\ Physical Review A (Atomic, Molecular and Optical Physics)
}

DOI:

10.1103/PhysRevA.56.4960

1997

Link to publication

Citation for published version (APA):

Antoine, P., Milosevic, D. B., L'Huillier, A., Gaarde, M. B., Salieres, P., \& Lewenstein, M. (1997). Generation of attosecond pulses in macroscopic media. Physical Review A (Atomic, Molecular and Optical Physics), 56(6), 4960-4969. https://doi.org/10.1103/PhysRevA.56.4960

\section{Total number of authors:}

6

\section{General rights}

Unless other specific re-use rights are stated the following general rights apply:

Copyright and moral rights for the publications made accessible in the public portal are retained by the authors and/or other copyright owners and it is a condition of accessing publications that users recognise and abide by the legal requirements associated with these rights.

- Users may download and print one copy of any publication from the public portal for the purpose of private study or research.

- You may not further distribute the material or use it for any profit-making activity or commercial gain

- You may freely distribute the URL identifying the publication in the public portal

Read more about Creative commons licenses: https://creativecommons.org/licenses/

Take down policy

If you believe that this document breaches copyright please contact us providing details, and we will remove

access to the work immediately and investigate your claim.
} 


\title{
Generation of attosecond pulses in macroscopic media
}

\author{
Philippe Antoine, ${ }^{1,2}$ Dejan B. Milošević, ${ }^{1,3}$ Anne L'Huillier, ${ }^{4}$ Mette B. Gaarde, ${ }^{4,5}$ Pascal Salières, ${ }^{2}$ and Maciej Lewenstein ${ }^{2}$ \\ ${ }^{1}$ Laboratoire de Physique Atomique et Moléculaire, Université Catholique de Louvain, 2 Chemin du Cyclotron, \\ B-1348 Louvain-la-Neuve, Belgium \\ ${ }^{2}$ Commissariat à l'Energie Atomique, DSM DRECAM SPAM Centre d'Etudes de Saclay, 91191 Gif-sur-Yvette, France \\ ${ }^{3}$ Department of Physics, Faculty of Science and Mathematics, University of Sarajevo, Vilsonovo šetalište 9, 71000 Sarajevo, Bosnia \\ and Faculty of Mechanical Engineering, Department of Precise Mechanics and Optics, University of Sarajevo, \\ Vilsonovo šetalište 9, 71000 Sarajevo, Bosnia \\ ${ }^{4}$ Department of Physics, Lund Institute of Technology, S-221 00 Lund, Sweden \\ ${ }^{5}$ Niels Bohr Institute, Orsted Laboratory, 2100 Copenhagen, Denmark
}

(Received 13 June 1997)

\begin{abstract}
We describe theoretically the generation of ultrashort (subfemtosecond) pulses using high-order harmonics of a laser pulse with a time-dependent degree of ellipticity. The single-atom response is calculated by using a low-frequency strong-field approximation. Propagation effects are taken into account using a method going beyond the slowly varying envelope approximation. Propagation modifies significantly the results obtained in the single-atom response and, in certain conditions, makes the generation of one attosecond pulse possible. We discuss prospects for the observation of these ultrashort pulses.
\end{abstract}

[S1050-2947(97)09411-0]

PACS number(s): $32.80 . \mathrm{Rm}, 42.65 . \mathrm{Ky}$

\section{INTRODUCTION}

High-order harmonic generation (HG) is a rapidly developing area in the field of intense laser-atom interactions. One of the most exciting applications of these processes is the generation of pulses of electromagnetic radiation of very short duration (a few hundred attoseconds). This new topic has gained enormous interest since it may open the way to attosecond spectroscopy and, generally speaking, attosecond physics [1].

Several proposals for subfemtosecond pulse generation have been put forward during the last few years. They all involve high-order harmonic generation, mostly from gaseous media. There are essentially two types of proposals: (a) those that rely on phase locking between consecutive harmonics [2-4], and (b) those that concern single harmonics [5-7].

The first type of proposals is based on the observation that the harmonic spectrum consists of a plateau with peaks equally spaced in frequency and with nearly equal amplitudes. Farkas and Toth, and Harris et al. [2] speculated that, by filtering out $N$ harmonics from the plateau, one could obtain a train of intense ultrashort pulses of duration $T / 2 N$, where $T$ is the laser period, provided the harmonics were locked in phase. Antoine et al. [3] showed that, though it does not turn out to be true on the level of individual atoms, the emission by the macroscopic medium does consist of a regular train of short pulses thanks to the time filtering induced by macroscopic propagation effects. Furthermore, Corkum and his collaborators [4] suggested that by using laser fields with a time-modulated degree of ellipticity, it should become possible to generate a single attosecond pulse thus, in a way, achieving extraction of a pulse from the train of attosecond pulses obtained with linear polarization. This idea is based on the strong sensitivity of the harmonic conversion efficiency to the degree of ellipticity of the driving laser field $[8,9]$. Several methods have been proposed to modulate in time the laser degree of ellipticity $[4,10]$.

Schafer and Kulander, and Christov et al. [5,6] proposed to use very short fundamental pulses (below $25 \mathrm{fs}$ ) to generate very short harmonic pulses, especially for the harmonics in the cutoff region. Further compression can be achieved by compensating the dynamically induced (practically linear) chirp of the harmonics $[7,11]$.

Finally, it is worth mentioning another scheme based on high-order harmonics produced by laser pulses interacting with solid targets [12-15]. Using a simple model for the oscillating plasma surface, Rzążewski et al. [16] showed that when a Ti-sapphire laser pulse is normally incident on a surface, the reflected signal has the form of a train of ultrashort pulses (of duration $\simeq 100$ attoseconds). This result is rather interesting, since, on one hand, the harmonic spectrum is monotonically decreasing and does not exhibit any plateau [17], while, on the other hand, the production of those ultrashort pulses does not require any filtering.

It should be stressed that most of the above-discussed schemes have been considered only for single atoms. All of these proposals require a careful study of macroscopic effects, since propagation and phase matching in the macroscopic medium may significantly alter the single-atom signal. The purpose of the present work is to investigate in detail, on a single-atom level, as well as including propagation, the feasibility of the proposal from Corkum et al. [4] to use laser pulses with a time-modulated degree of ellipticity. We discuss under which conditions a single attosecond pulse can be generated, and which power can be obtained.

The plan of the paper is the following. In Sec. II we present our theoretical approach. The single-atom response is described using our quantum mechanical theory [18] valid in the tunneling limit. Propagation equations are solved by using an approximation that goes beyond the slowly varying envelope description, in order to account for a rapidly vary- 
ing degree of ellipticity for the fundamental field. In Sec. III we discuss our numerical results and in Sec. IV the effects of dispersion and ionization. The general conclusion of our work, presented in Sec. V, is that the scheme of Corkum and co-workers does work in a macroscopic medium, provided the duration of the fundamental laser pulse is short enough (below $30 \mathrm{fs}$ ). It is in fact the interplay between single-atom response and phase-matching effects that makes possible the generation of one attosecond pulse.

\section{THEORETICAL DESCRIPTION}

We first discuss the case where depletion due to ionization and dispersion is neglected. We will discuss in Sec. IV how the theory can be extended to (partially) ionized media.

\section{A. Single-atom response}

The details of our theory can be found in Refs. [18-20]. Here we quote only the main results. Atomic units are used in this paragraph. The dipole moment of an atom $\vec{x}(t)$ in the strong electric field of a laser of frequency $\omega$ is calculated by evaluating the integral:

$$
\begin{aligned}
\vec{x}(t)= & i \int_{0}^{\infty} d \tau\left(\frac{\pi}{\nu+i \tau / 2}\right)^{3 / 2} \vec{d} *\left[\vec{p}_{s}-\vec{A}(t)\right] \\
& \times \exp \left[-i S\left(\vec{p}_{s}, t, \tau\right)\right] \overrightarrow{\mathcal{E}}(t-\tau) \cdot \vec{d}\left[\vec{p}_{s}-\vec{A}(t-\tau)\right]+\text { c.c. }
\end{aligned}
$$

$\nu$ is a positive regularization constant, $\vec{A}(t)$ denotes the vector potential of the electromagnetic field, $\overrightarrow{\mathcal{E}}(t)=-\partial \vec{A}(t) / \partial t$ is the electric field (elliptically polarized, in general). $S(\vec{p}, t, \tau)=\int_{t-\tau}^{t} d t^{\prime \prime}\left\{\left[\vec{p}-\vec{A}\left(t^{\prime \prime}\right)\right]^{2} / 2+I_{p}\right\}$ ( $I_{p}$ denoting the ionization potential) is the quasiclassical action, describing the motion of an electron moving in the laser field with a constant canonical momentum $\vec{p}$. Equation (1) is a generalized Landau-Dyhne formula, obtained by using a saddle-point method to integrate over all possible values of the momentum $\vec{p}$ with which the electron is born in the continuum. The integration variable $\tau$ represents the possible return times of the electron, i.e., the times it spends in the continuum between the moments of tunneling from the ground state to the continuum and recombination back to the ground state. The saddle-point value of the momentum (for which the quasiclassical action is stationary), and which enters in Eq. (1), is $\vec{p}_{s}=\vec{p}_{s}(t, \tau)=\int_{t-\tau}^{t} d t^{\prime \prime} \vec{A}\left(t^{\prime \prime}\right) / \tau$. Finally, the field-free dipole transition element from the ground state to the continuum state characterized by the momentum $\vec{p}$ can be approximated by

$$
\vec{d}(\vec{p})=i \frac{2^{7 / 2} \alpha^{5 / 4}}{\pi} \frac{\vec{p}}{\left(\vec{p}^{2}+\alpha\right)^{3}},
$$

with $\alpha=2 I_{p}$, for the case of hydrogenlike atoms and transitions from $s$ states [21]. Since the propagation equations are expressed in the frequency domain, in the general case, we need to calculate the Fourier transform $\vec{x}_{\Omega}$ of $\vec{x}(t)$ for a continuous range of frequencies. When the field is periodic, of course, the set of frequency components becomes discrete. In the case of linear polarization, and long pulses, for which the slowly varying envelope can be applied, the frequency components reduce to the harmonics of the laser field.

\section{B. Propagation equations beyond the slowly varying approximation}

We start from the general wave equation describing the propagation of an electromagnetic field $\overrightarrow{\mathcal{E}}(\vec{r}, t)$ in an isotropic, globally neutral, nonmagnetic, dielectric medium, characterized by an electronic polarization $\overrightarrow{\mathcal{P}}(\vec{r}, t)$ [here we use Systeme International (SI) units]:

$$
\nabla^{2} \overrightarrow{\mathcal{E}}(\vec{r}, t)-\frac{1}{c^{2}} \frac{\partial^{2} \overrightarrow{\mathcal{E}}(\vec{r}, t)}{\partial t^{2}}=\frac{1}{\epsilon_{0} c^{2}} \frac{\partial^{2} \overrightarrow{\mathcal{P}}(\vec{r}, t)}{\partial t^{2}}
$$

The Fourier transform of this equation reads

$$
\nabla^{2} \overrightarrow{\mathcal{E}}_{\Omega}(\vec{r})+\frac{\Omega^{2}}{c^{2}} \overrightarrow{\mathcal{E}}_{\Omega}(\vec{r})=-\frac{\Omega^{2}}{\epsilon_{0} c^{2}} \overrightarrow{\mathcal{P}}_{\Omega}(\vec{r})
$$

The polarization $\overrightarrow{\mathcal{P}}_{\Omega}(\vec{r})$ at frequency $\Omega$ consists of a linear component, characterizing the linear response of the medium at that frequency, and a nonlinear component, which, in the absence of depletion, is simply proportional to $\vec{x}_{\Omega}(\vec{r})$, the Fourier component of the atomic dipole moment, calculated for the (complex) amplitude of the fundamental field at the position $\vec{r}$ (we neglect wave-mixing processes involving absorption of the generated fields). We now use the paraxial approximation which assumes that the laser and generated fields propagate essentially like a plane wave. We introduce the envelopes $\vec{E}_{\Omega}(\vec{r})=\overrightarrow{\mathcal{E}}_{\Omega}(\vec{r}) \exp \left(-i k_{\Omega} z\right)$ and $\vec{P}_{\Omega}(\vec{r})$ $=\overrightarrow{\mathcal{P}}_{\Omega}(\vec{r}) \exp \left(-i k_{\Omega} z\right)$, with $k_{\Omega}=\Omega / c$, in the absence of dispersion. $z$ denotes the coordinate on the propagation axis. We assume that $\vec{E}_{\Omega}(\vec{r})$ and $\vec{P}_{\Omega}(\vec{r})$ vary slowly in the coordinate $z$ over the field wavelength. The linear part of the polarization cancels out with terms in the left-hand side in Eq. (4) and we obtain the following equation for the envelopes:

$$
\nabla_{\perp}^{2} \vec{E}_{\Omega}(\vec{r})+2 i k_{\Omega} \frac{\partial \vec{E}_{\Omega}(\vec{r})}{\partial z}=-\frac{\Omega^{2}}{\epsilon_{0} c^{2}} \vec{P}_{\Omega}^{\mathrm{nl}}(\vec{r}),
$$

where $\vec{P}_{\Omega}^{\mathrm{nl}}(\vec{r})$ is the nonlinear polarization envelope. It is worth stressing that, by solving Eq. (5) for a continuous range of frequencies, we go beyond the adiabatic (slowly varying envelope) approximation in time. In particular, we account for the time dependence of the fundamental in a nonadiabatic way.

To obtain the time dependence of the total field $\overrightarrow{\mathcal{E}}(\vec{r}, t)$, one has to Fourier transform back to the time domain:

$$
\overrightarrow{\mathcal{E}}(\vec{r}, t)=\frac{1}{2 \pi} \int \vec{E}_{\Omega}(\vec{r}) \exp \left[-i\left(\Omega t-k_{\Omega} z\right)\right] d \Omega .
$$

To investigate the response of a certain range of frequencies, say between $\Omega_{\min }$ and $\Omega_{\max }$, one simply limits the integration to that particular range. Finally, the integrated temporal signal is equal to $\int|\overrightarrow{\mathcal{E}}(\vec{r}, t)|^{2} 2 \pi r d r$. 


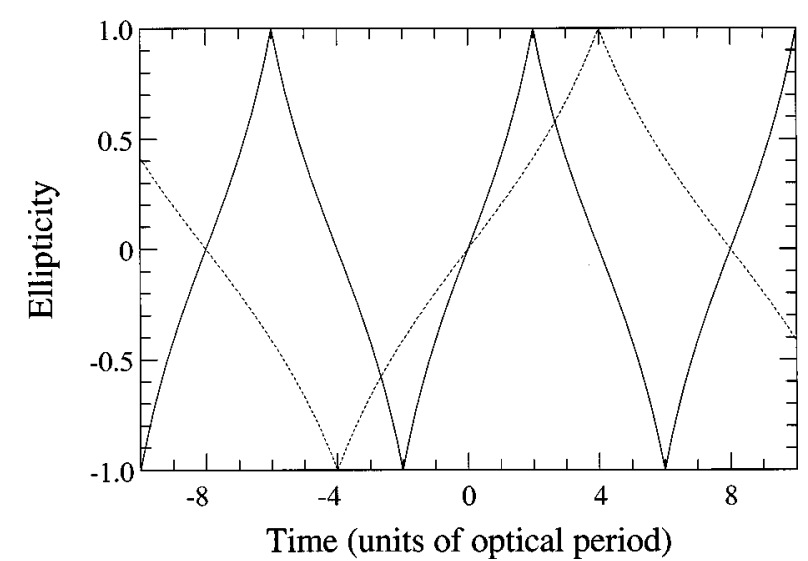

FIG. 1. Time dependence of the ellipticity for $\Delta=\bar{\omega} / 32$ (dashed line) and $\Delta=\bar{\omega} / 16$ (solid line).

\section{Electromagnetic field with a modulated degree of ellipticity}

A simple way to obtain a laser field with a time-dependent degree of ellipticity, proposed by Corkum et al. [4], is to use two cross-polarized fields with slightly different frequencies:

$$
\overrightarrow{\mathcal{E}}_{0}(t)=\mathcal{E}_{0} f(t)\left(\cos \left(\omega_{1} t\right), \cos \left(\omega_{2} t\right), 0\right),
$$

where $\mathcal{E}_{0}$ is the field amplitude, $f(t)$ is the pulse envelope, and $\omega_{1}$ and $\omega_{2}$ are the two laser frequencies. We introduce $\bar{\omega}=\left(\omega_{1}+\omega_{2}\right) / 2$ and $\Delta=\left(\omega_{2}-\omega_{1}\right) / 2$. The phase difference between the two perpendicular components varies in time, and is equal to $2 \Delta t$. The total field is elliptically polarized, with a time-varying ellipticity. The axes of the ellipse are oriented at $\pm 45^{\circ}$ with respect to the $x, y$ axes. The instantaneous value of the ellipticity is $\epsilon(t)=\tan (\Delta t)$ for $-\pi / 4 \leqslant \Delta t \leqslant \pi / 4$, and $\epsilon(t)=\cot (\Delta t)$ for $\pi / 4 \leqslant \Delta t \leqslant 3 \pi / 4$ modulo $\pi$. The sign of $\epsilon(t)$ represents the sense of rotation of the ellipse (helicity). In Fig. 1, we plot $\epsilon(t)$ for two choices of $\Delta=\bar{\omega} / 32$ (solid line) and $\Delta=\bar{\omega} / 16$ (dashed line). Since $\Delta$ is commensurable with $\bar{\omega}$, the ellipticity $\epsilon(t)$ is a periodic function with a period equal to 16 and 8 optical periods, respectively (one optical period is $T=2 \pi / \bar{\omega}$ ). In these cases, assuming that the pulse envelope changes on a much slower time scale, so that the intensity is approximately constant over this time range, the response of a single atom submitted to a field given by Eq. (7) will also be periodic with the same period as the ellipticity.

\section{NUMERICAL RESULTS}

\section{A. Single-atom response}

Most of our calculations, in particular those for the singleatom emission, have been performed for a constant field intensity $\left[I=2\left|\mathcal{E}_{0}\right|^{2}, f(t)=1\right.$ in Eq. (7)], i.e., as if the laser pulse were infinitely long. This implies that the range of frequencies necessary to describe the response of an atom to the field defined in Eq. (7) is discrete provided that $\Delta$ is commensurable with $\bar{\omega}$. Consider a process where $n_{1}$ photons are absorbed from the first field and $n_{2}$ from the second field. Parity implies that $n_{1}+n_{2}$ is odd, and so is $n_{2}-n_{1}$. The frequency of the emission is $n_{1} \omega_{1}+n_{2} \omega_{2}$ $=\left(n_{1}+n_{2}\right) \bar{\omega}+\left(n_{2}-n_{1}\right) \Delta$. In our case, this implies that the spectrum consists of odd harmonics of $\Delta$ since $\bar{\omega} / \Delta$ is even.

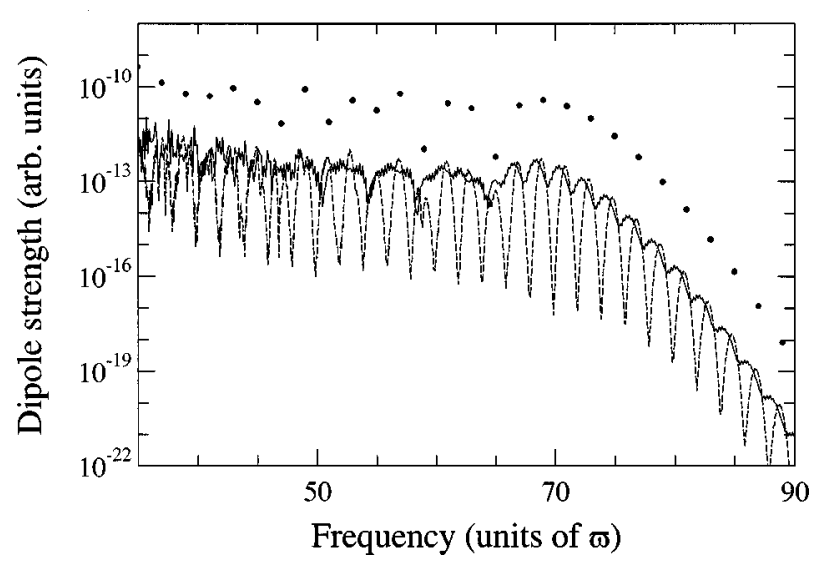

FIG. 2. Frequency spectrum for a single neon atom for $\Delta=0$ (dots, linear polarization), $\Delta=\bar{\omega} / 32$ (dashed line), and $\Delta=\bar{\omega} / 16$ (solid line). The laser intensity is $4 \times 10^{14} \mathrm{~W} / \mathrm{cm}^{2}$, and the wavelength $825 \mathrm{~nm}$.

In Fig. 2, we present three single-atom spectra obtained in neon. In all cases, the peak intensity is chosen to be $4 \times 10^{14} \mathrm{~W} / \mathrm{cm}^{2}$, and the wavelength $\lambda=2 \pi c / \bar{\omega}, 825 \mathrm{~nm}$. The dots represent the results obtained for linear polarization. The spectrum then consists of odd harmonics of the fundamental. The solid and dashed lines have been obtained for two (infinitely long) pulses, defined as in Eq. (7) for $\Delta=\bar{\omega} / 16$ and $\Delta=\bar{\omega} / 32$, respectively. The peaks at the odd harmonic frequencies of $\Delta$ have been joined by a line for the sake of clarity. When $\Delta=\bar{\omega} / 32$, the spectrum shows maxima at the (odd) harmonic frequencies of $\bar{\omega}$. This is because the variation of the ellipticity is relatively slow compared to the optical cycle and some periodicity at the mean frequency is conserved. In contrast, when $\Delta=\bar{\omega} / 16$, the harmonic structure is almost lost. The spectrum is more or less continuous in the plateau region with a (slightly shifted) harmonic structure in the cutoff region. The results obtained with a timevarying degree of polarization are about two orders of magnitude lower than those with linear polarization. This is because the time during which emission occurs is limited to about $1 / 8$ of that for the case of linear polarization (see below), so that the harmonic strength is approximately a factor of 64 below.

In the present work, we want to examine the temporal response of a limited frequency range (corresponding to a few, typically ten, harmonics in the linear polarization case). We consider the filtered signal:

$$
\left|\vec{x}_{F}(t)\right|^{2}=\left|\sum_{\Omega_{\min } \leqslant \Omega \leqslant \Omega_{\max }} \vec{x}_{\Omega} \exp (-i \Omega t)\right|^{2},
$$

keeping only the frequency components $\Omega_{\min }=41 \bar{\omega}$ $\leqslant \Omega \leqslant \Omega_{\max }=61 \bar{\omega}[22]$. This temporal signal is plotted in Fig. 3 over three optical periods in the three cases mentioned above: (a) linear polarization, (b) time-dependent elliptical polarization for $\Delta=\bar{\omega} / 32$, (c) and (d) time-dependent elliptical polarization for $\Delta=\bar{\omega} / 16$. In the case of linear polarization [Fig. 3(a)], the time profile is rather complex. This result has been discussed in detail in Ref. [3]. The harmonics in the plateau region generated by a single atom are not locked in phase, since they result from quantum interferences between probability amplitudes corresponding to various paths for the 

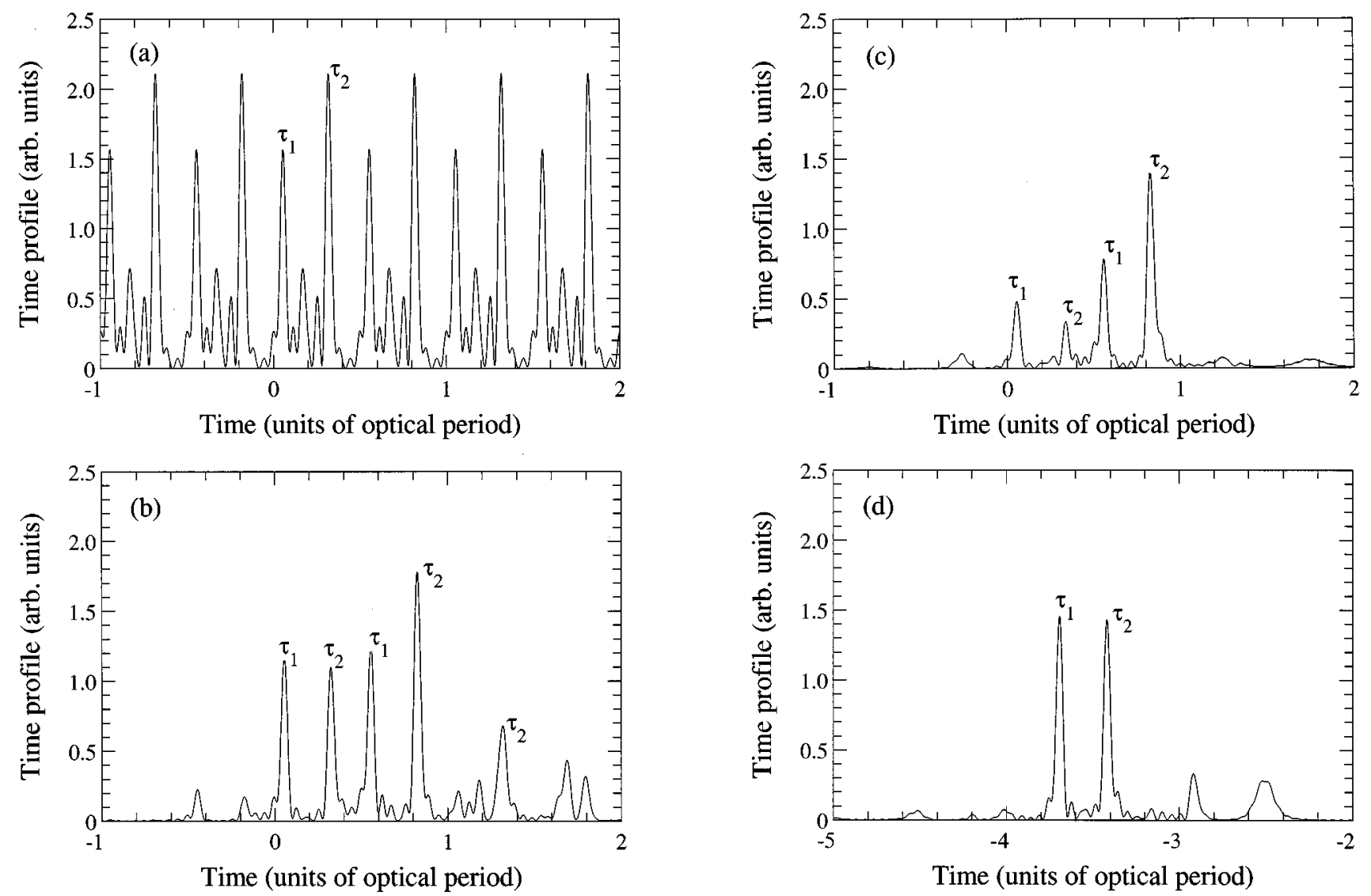

FIG. 3. Time profile $\left|\vec{x}_{F}(t)\right|^{2}$ calculated for a single neon atom and composed of the Fourier components with $\Omega_{\min }=41 \bar{\omega} \leqslant \Omega \leqslant \Omega_{\max }=61 \bar{\omega}$. The laser intensity is $4 \times 10^{14} \mathrm{~W} / \mathrm{cm}^{2}$, and the wavelength $825 \mathrm{~nm}$. (a) Linear polarization, (b) time-dependent elliptical polarization with $\Delta=\bar{\omega} / 32$, (c) and (d) time-dependent elliptical polarization with $\Delta=\bar{\omega} / 16$.

generation, associated to different electron trajectories in the continuum [1]. As a result, the time profile of the filtered signal consists of a complex train of pulses, with several peaks per half cycle. The dominant peaks in the train can be associated directly to the electronic trajectories that occur with the dominant probability amplitudes. In particular, the largest one corresponds to a trajectory lasting about one cycle (return time $\tau_{2}$ ), whereas the next largest corresponds to a shorter one, lasting about half a cycle (return time $\tau_{1}$ ). The smaller peaks are associated to longer trajectories.

In the case of the time-dependent elliptical polarization with $\Delta=\bar{\omega} / 32$ [Fig. 3(b)], the instantaneous field [Eq. (7)] becomes linear at the times $t_{\operatorname{lin}}=\ldots,-8 T, 0 T, 8 T, \ldots$ ( $T$ is the mean optical period). Due to the dramatic decrease of the harmonic generation efficiency with increasing ellipticity, the harmonics are now generated in the form of bursts close to the times $t_{\text {lin }}$. Figure 3(b) shows the temporal signal close to $t=0$. The emission peaks are mostly after the times $t_{\text {lin }}$. This is because there is a time lag, depending on the electron trajectory, between tunneling and harmonic emission. For $t_{\text {lin }}=0$, tunneling takes place close to $t=-0.5 T, 0 T, 0.5 T$, i.e., when the field is close to its maximum, and the most efficient emission occurs within the first period afterwards, i.e., between $t=0 T$ and $t=1.5 T$. Let us consider the five highest peaks in Fig. 3(b). The first $(t=0.05 T)$ and third $(t=0.55 T)$ ones correspond to "short" trajectories in the linear polarization case [see Fig. 3(a)], lasting about half a cycle. The tunneling times for these trajectories are approxi- mately $t=-0.45 T$ and $t=0.05 T$. The second $(t=0.25 T)$, fourth $(t=0.75 T)$, and fifth $(t=1.25 T)$ peaks correspond to "long" trajectories in the linear polarization case, lasting slightly less than one cycle. The present time profile confirms, at least qualitatively, these considerations based on stationary phase arguments [23]. The amplitudes of the first and the third peaks can be comparable only if the mean degrees of ellipticity during the corresponding electron trajectories are alike. This implies that the return times should be close to $0.5 T$. The presence of the fifth peak $(t=1.25 T)$, while no significant peak appears at $t=1.05 T$, indicates that the peaks at $t=0.25 T, 0.75 T, 1.25 T, \ldots$ correspond to trajectories with longer return times than the trajectories characterizing the first and third peaks in Fig. 3(b). Furthermore, the time profile clearly indicates that the wave packet recollides several times with the core. The consequence of this quasiperiodicity is a spectrum with well defined harmonics $[24,25]$.

In the case of the time-dependent elliptical polarization with $\Delta=\bar{\omega} / 16$ [Figs. 3(c),(d)], the instantaneous field [Eq. (7)] becomes linear at the times $t_{\text {lin }}$ $=\ldots,-8 T,-4 T, 0 T, 4 T, \ldots$. Figure 3(c) shows the harmonic bursts close to the time $t_{\text {lin }}=0 T$. They are very similar to those of Fig. 3(b), except that the difference between the peaks (in particular between the second and fourth) is more pronounced, owing to the more rapidly varying degree of ellipticity. Note that the bursts close to $t_{\text {lin }}=\ldots,-8 T, 0 T, 8 T, \ldots$ are all identical, but different from 

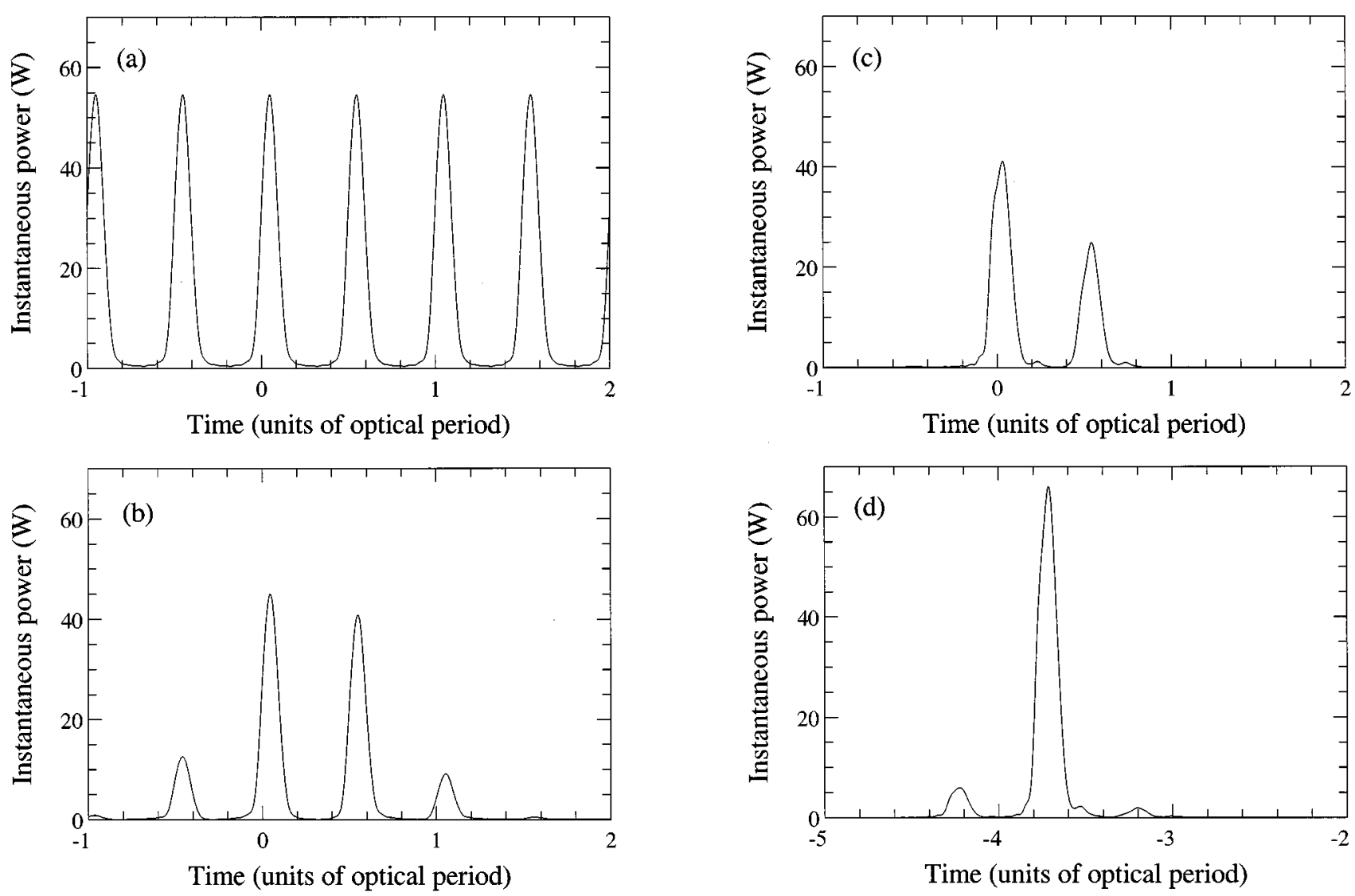

FIG. 4. Same as Fig. 3 for the macroscopic response calculated for a $1 \mathrm{~mm}$ wide atomic jet located $2 \mathrm{~mm}$ after the focus. The laser intensity is equal to $4 \times 10^{14} \mathrm{~W} / \mathrm{cm}^{2}$ at the center of the jet.

those at $t_{\text {lin }}=\ldots,-4 T, 4 T, 12 T, \ldots$. (In the $\Delta=\bar{\omega} / 32$ case, similarly, the bursts at $-8 T, 8 T$ are different from those at $-16 T, 0 T, 16 T$.) The reason is that the periodicity of the field defined in Eq. (7) for $\Delta=\bar{\omega} / 16$ is $8 T$ and not $4 T$. The electric field is maximum at $t=-8 T, 0 T, 8 T, \ldots$ whereas it vanishes at $t=-4 T, 4 T, \ldots$. Around $t=-4 T, 4 T, \ldots$, the maxima of the electric field are thus shifted by $T / 4$ with respect to the surroundings of $t=-8 T, 0 T, 8 T, \ldots$. The trajectories are also shifted by the same value. In Fig. 3(d), the remaining peaks correspond to trajectories occurring around $t=-4 T$ when the polarization is close to linear and thus the mean degree of ellipticity is minimum.

We stress that our results are different from those of Ref. [4], where the single-atom response consisted of a regular train of attosecond pulses, and one of these pulses was selected using a fundamental pulse with a time-varying degree of ellipticity. Here we consider the case when the harmonics that contribute to the signal are deep in the plateau region, where the generation is most efficient. This is the regime dominated by quantum interference effects, for which the response of the atom to a linearly polarized field is irregular [Fig. 3(a)]. It is not surprising that, in this case, the timedependent polarization does not enable the selection of a single attosecond pulse per $8 T$ [Fig. 3(b)] or per $4 T$ [Figs. $3(\mathrm{c}),(\mathrm{d})]$. It selects in general two pulses per half cycle, i.e., about four pulses in total in the vicinity of $t=t_{\text {lin }}$. Even when the degree of ellipticity changes very rapidly $(\Delta=\bar{\omega} / 16)$, some $T / 2$ periodicity appears around $t_{\text {lin }}=-8 T, 0 T, 8 T, \ldots$. As a consequence, it should be pos- sible to distinguish some harmonic structure in the spectrum, even with a poor contrast. We attribute the smearing of the harmonics in the spectrum (Fig. 2) to an additional shift, dependent on the return time, induced by the time-dependent degree of ellipticity. The structure of the cutoff is preserved because there only one return time leads to harmonic generation. Even if the mechanism is probably different, the smearing of the spectrum in the plateau obtained for an infinitely long pulse, but for a rapidly varying degree of ellipticity, actually resembles that obtained for a short pulse and linear polarization $[5,6,25,26]$.

\section{B. Many-atom response}

We first present the results for a constant field intensity. To describe the many-atom response to the field described in Eq. (7), it is necessary to know the Fourier components of the atomic dipole moment, calculated for the fundamental field $\left(\mathcal{E}_{0}(\vec{r}) \cos \left[\omega_{1} t+\phi(\vec{r})\right], \mathcal{E}_{0}(\vec{r}) \cos \left[\omega_{2} t+\phi(\vec{r})\right], 0\right)$, whose amplitude and phase vary in the nonlinear medium. We here assume that focusing of the two fields is the same (given the small frequency difference), which implies that the amplitude and phase of the two components are the same. How to obtain the components $\vec{x}_{\Omega}(\vec{r})$ from those calculated previously for a field defined as in Eq. (7) is explained in detail in the Appendix.

The results presented below for the macroscopic response have been obtained under the following conditions. The $(825$ $\mathrm{nm}$ ) fundamental field is supposed to be Gaussian with a 
confocal parameter $b=5 \mathrm{~mm}$. The intensity is chosen to be $6.56 \times 10^{14} \mathrm{~W} / \mathrm{cm}^{2}$ at focus. A $1 \mathrm{~mm}$ wide jet of neon atoms is located $2 \mathrm{~mm}$ after the focus so that the peak intensity at the center of the jet is $4 \times 10^{14} \mathrm{~W} / \mathrm{cm}^{2}$.

We calculate as before the filtered time profiles composed of the sum of the Fourier components of the propagated field for $\Omega_{\min }=41 \bar{\omega} \leqslant \Omega \leqslant \Omega_{\max }=61 \bar{\omega}$. The results are shown in Figs. 4(a) $-4(d)$, for linear polarization (a), time-dependent elliptical polarization with $\Delta=\bar{\omega} / 32$ (b), and time-dependent elliptical polarization with $\Delta=\bar{\omega} / 16$, (c) and (d). The unit on the vertical axis is an instantaneous power (square of the electric field amplitude integrated over the plane perpendicular to the propagation axis). Note that these results probably underestimate the harmonic intensities by a factor of the order of 5-10 [21].

In the linear polarization case, the time profile consists of a train of pulses of duration 300 attoseconds (as). As explained in Ref. [3], propagation selects only one of the two dominant trajectories: In the focusing conditions chosen here, it is the trajectory with the short return time [compare Figs. 3(a) and 4(a)]. The reason for the selection is that the phase-matching conditions are different for the two trajectories (since they are associated to different phase variations) and are not fulfilled simultaneously. As shown in Figs. 4(b)4(d), this conclusion is also true when the degree of ellipticity varies in time. Essentially two peaks are selected in Figs. 4(b) and 4(c). These peaks are the first and third peaks of the series of four peaks obtained in Figs. 3(b) and 3(c), which correspond to the short trajectories. Finally, Fig. 4(d) shows selection of only a single attosecond pulse. Note that the instantaneous power is about the same as in the linear polarization case. The number of photons contained in one of these pulses can be estimated to be about 1000 .

The spectra obtained in the three cases $(\Delta=0$, i.e., linear polarization, $\Delta=\bar{\omega} / 32$, and $\Delta=\bar{\omega} / 16)$ and in the same geometrical conditions (jet positioned $2 \mathrm{~mm}$ after the focus) are shown in Fig. 5(a). We use the same conventions as in Fig. 2. The number of photons is estimated by assuming a square pulse of duration $25 \mathrm{fs}$. In all cases, the spectra are significantly more regular than in the single-atom response. This is because phase matching selects essentially one trajectory (in the conditions chosen, the shortest one), so that quantum interferences vanish. Interestingly, in the $\Delta=\bar{\omega} / 16$ case, the harmonic structure, which had disappeared in the singleatom response, is clearly present. To better understand this effect, we have calculated the same spectra in different geometrical conditions, when the gas jet is positioned at the focus, keeping the same laser intensity at the center of the gas jet $\left(I=4 \times 10^{14} \mathrm{~W} / \mathrm{cm}^{2}\right)$. In these conditions, phase matching favors the other dominant electron trajectory [3], corresponding to the longer return times of the order of the optical cycle. The results are shown in Fig. 5(b) for the three polarization cases. The spectrum obtained for $\Delta=\bar{\omega} / 16$ presents a remarkable property: it consists of a series of (broad) peaks centered at the even harmonics of the fundamental frequency (a small frequency shift appears already in the spectrum corresponding to the $\Delta=\bar{\omega} / 32$ case). This is again a very interesting effect of time filtering due to the propagation. It allows us to understand the origin and composition of the single-atom emission spectrum. The frequencies close to the even harmonic ones are associated to the longer trajec-
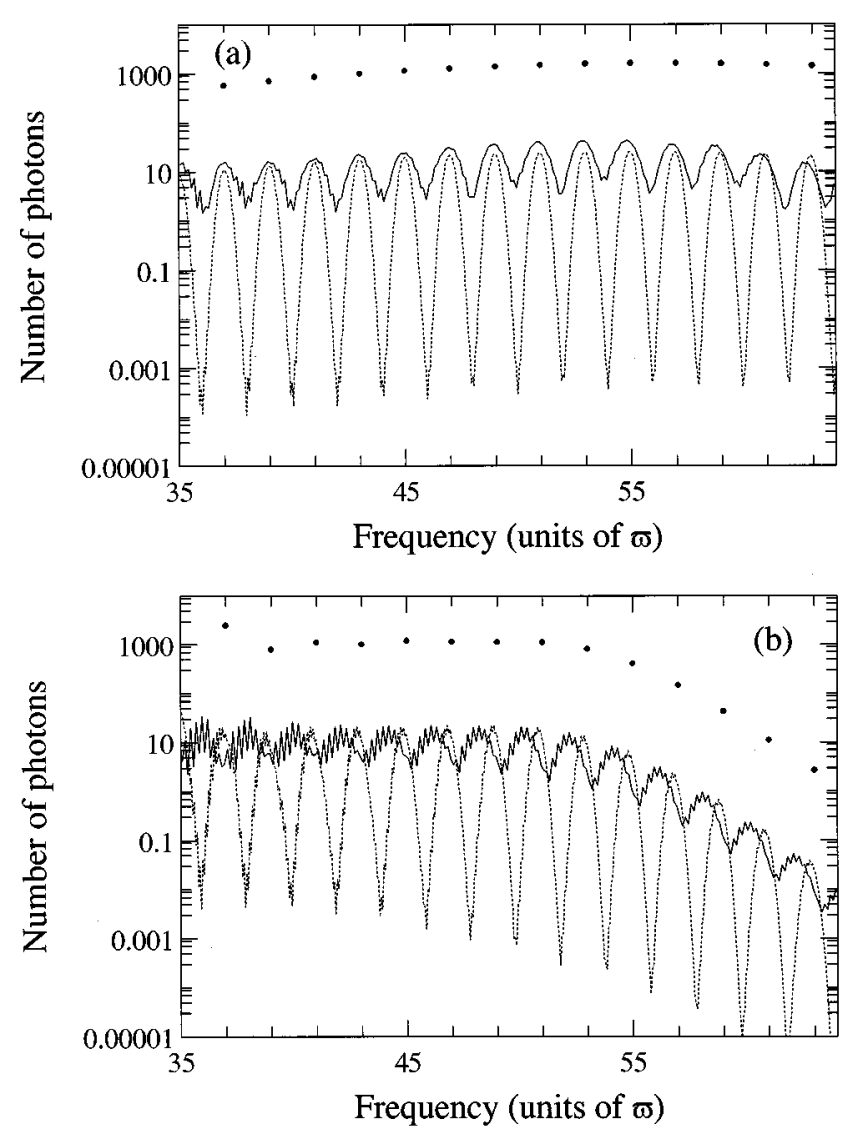

FIG. 5. Same as Fig. 2 for the macroscopic response. The laser pulse is square with a $25 \mathrm{fs}$ width. (a) The $1 \mathrm{~mm}$ wide jet is located $2 \mathrm{~mm}$ after the focus; (b) the jet is centered at the focus.

tory, whereas the ones close to the odd harmonic frequencies correspond to the shorter trajectory. In other words, the harmonic frequencies are shifted depending on the return time corresponding to the electron trajectory. This property has an interesting consequence from the experimental point of view. The observation of the variation of the positions of the harmonic peaks in the spectrum as a function of the geometrical conditions (position of the atomic medium relative to the laser focus) provides an (indirect) signature of the emission of a single, or perhaps two attosecond pulses.

\section{Influence of the pulse shape}

To take into account rigorously the influence of the laser pulse duration, as described in Sec. II, it is necessary to calculate the single-atom response for a laser pulse whose degree of polarization and envelope vary in time, Fourier transform the time-dependent dipole moment, propagate the different frequency components, and inverse Fourier transform to obtain the time-dependent response of the medium. In contrast to the case of an infinitely long pulse, there is no periodicity and the range of frequencies is continuous. In the present work, however, we treat the pulse envelope as a slow process in comparison to the other time variation and we perform a slowly varying envelope approximation for the pulse envelope. This is a crude approximation since, in the numerical example chosen ( 25 fs pulse width) the pulse envelope variation is actually comparable to that of the degree of ellipticity (for the $\Delta=\bar{\omega} / 16$ case, the periodicity is $8 T$, 

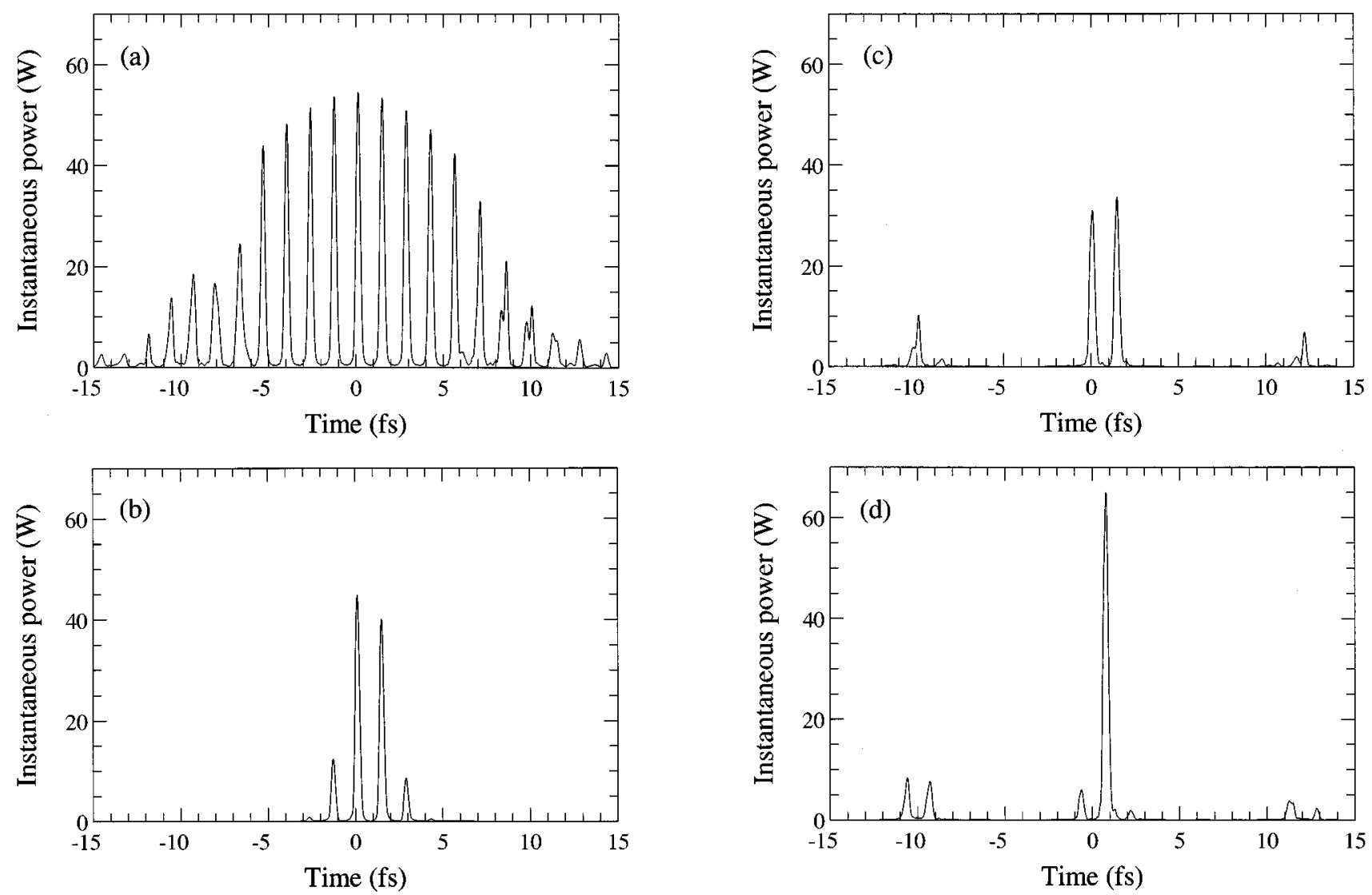

FIG. 6. Same result as in Fig. 4, for a Gaussian laser pulse of 25 fs duration.

i.e., $22 \mathrm{fs}$ ). Here our aim is only to estimate how short the pulse width should be to enable the generation of only one "attosecond" pulse.

The numerical method consists of assuming the laser intensity to be constant over a given time step $I(t)=I_{k}, t_{k-1}<t<t_{k}$ and of calculating the time-dependent response of the nonlinear medium for this series of (constant) peak intensities $I_{k}$. The time-dependent response of the nonlinear medium to a laser pulse can then be easily reconstructed. Results obtained for a 25 fs laser pulse are shown in Fig. 6, for the same four cases as before: linear polarization (a), time-dependent elliptical polarization with $\Delta=\bar{\omega} / 32$ (b), and with $\Delta=\bar{\omega} / 16$, (c) and (d). In Fig. 6(d), we have introduced a phase shift of $\pi / 2$ in both components in Eq. (7), so that the photon emission occurring previously slightly after $t_{\text {lin }}=-4 T$ [see Figs. 3(d) and 4(d)] is shifted in time and is now centered at the top of the pulse. In the $\Delta=\bar{\omega} / 32$ case, the pulse duration is short enough so that only one burst of mainly two attosecond pulses is emitted. In the $\Delta=\bar{\omega} / 16$ case, there are three groups of short pulses, but the "sidebands" emitted at the beginning and end of the pulse have much lower power than the pulses emitted close to the top of the laser pulse. In the case shown in Fig. 6(d), the medium generates essentially one attosecond pulse of power $30 \mathrm{~W}$. This calculation shows that the laser pulse duration should be as short as 25 fs to enable the generation of a single attosecond pulse. This result proves that the proposal of Corkum and co-workers [4] works well for harmonics in the plateau, and enables efficient selection of a single attosecond pulse provided that the driving pulse is short enough.

\section{INFLUENCE OF IONIZATION EFFECTS}

We here concentrate on the effects due to ionization, depletion, and additional dispersion which are the most important affecting high-order harmonic generation in gases. In a general formalism, the depletion of the atomic medium due to ionization can be included via the Fourier transform of the electronic polarization:

$$
\begin{aligned}
\overrightarrow{\mathcal{P}}_{\Omega}(\vec{r}) & =\int \overrightarrow{\mathcal{P}}(\vec{r}, t) \exp (i \Omega t) d \Omega \\
& =\int \mathcal{N}(\vec{r}, t) \vec{x}(\vec{r}, t) \exp (i \Omega t) d \Omega \\
& =\left[\mathcal{N}_{\Omega} * \vec{x}_{\Omega}\right](\vec{r}),
\end{aligned}
$$

the sign $*$ denoting the convolution operation and $\mathcal{N}_{\Omega}$ is the Fourier transform of the density $\mathcal{N}(\vec{r}, t)$. Ionization, which depletes the medium density, perturbs not only the nonlinear response of the polarization but also the linear response at any frequency, i.e., it introduces a dispersion. Ionization is in general described by a rate so that the atomic density reads

$$
\mathcal{N}(\vec{r}, t)=\mathcal{N}_{a}(z) \exp \left(-\int_{-\infty}^{t} \Gamma\left(\vec{r}, t^{\prime}\right) d t^{\prime}\right),
$$

where $\mathcal{N}_{a}(z)$ is the initial atomic density in the gas jet which depends on the coordinate $z$ along the propagation axis. $\Gamma\left(\vec{r}, t^{\prime}\right)$ is the ionization rate due to the fundamental field at position $\vec{r}$ and time $t^{\prime}$. (Ionization due to the generated fields 


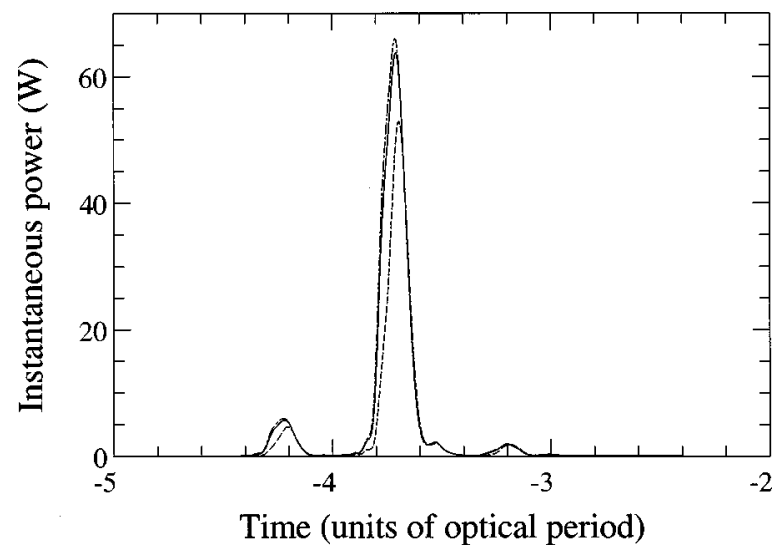

FIG. 7. Same result as in Fig. 4(d), with inclusion of ionization and dispersion effects for a square $25 \mathrm{fs}$ (solid line) and square 150 fs pulse (dashed line).

is negligible.) In our theoretical approach, we calculate $\Gamma$ as $2 \operatorname{Re}(\bar{\gamma})$, where $\bar{\gamma}$ is the time average of the complex decay rate $[27]$

$$
\begin{aligned}
\gamma(t)= & \int_{0}^{\infty} d \tau\left(\frac{\pi}{\nu+i \tau / 2}\right)^{3 / 2} \overrightarrow{\mathcal{E}}^{*}(t) \cdot \vec{d}^{*}\left[\vec{p}_{s}-\vec{A}(t)\right] \\
& \times \exp \left[-i S\left(\vec{p}_{s}, t, \tau\right)\right] \overrightarrow{\mathcal{E}}(t-\tau) \cdot \vec{d}\left[\vec{p}_{s}-\vec{A}(t-\tau)\right] .
\end{aligned}
$$

In the present work, we treat ionization as a slow perturbation, and we include it within a slowly varying envelope approximation, in a similar way as the pulse envelope (see preceding section). This allows us to separate the time variation of the density from that of the dipole moment [Eq. (9)]. The propagation equations [see Eq. (5)] take the form (see Ref. [20])

$$
\nabla_{\perp}^{2} \vec{E}_{\omega_{i}}(\vec{r}, t)+2 i k_{\omega_{i}} \frac{\partial \vec{E}_{\omega_{i}}(\vec{r}, t)}{\partial z}+2 k_{\omega_{i}} \delta k_{\omega_{i}}(\vec{r}, t) \vec{E}_{\omega_{i}}(\vec{r}, t)=0
$$

for the fundamental fields with frequencies $\omega_{i}, i=1,2$, and

$$
\begin{aligned}
\nabla_{\perp}^{2} & \vec{E}_{\Omega}(\vec{r}, t)+2 i k_{\Omega} \frac{\partial \vec{E}_{\Omega}(\vec{r}, t)}{\partial z}+2 k_{\Omega} \delta k_{\Omega}(\vec{r}, t) \vec{E}_{\Omega}(\vec{r}, t) \\
& =-\frac{\Omega^{2}}{\epsilon_{0} c^{2}} \vec{P}_{\Omega}^{\mathrm{nl}}(\vec{r}, t)
\end{aligned}
$$

for the generated fields at frequency $\Omega$. The time dependence in the equations refers to the slow time variation due to the pulse shape and to ionization effects. (It has not been accounted for in the Fourier transform.) The depletion is accounted for by taking $P_{\Omega}^{\mathrm{nl}}(\vec{r}, t)$ proportional to the atomic density. $\delta k_{\Omega}(\vec{r}, t)=-e^{2} \mathcal{N}_{e}(\vec{r}, t) / 2 m c \Omega$, with $e$ denoting the electron charge, $m$ its mass, and $\mathcal{N}_{e}(\vec{r}, t)=\mathcal{N}_{a}(z)-\mathcal{N}(\vec{r}, t)$ the electronic density, describes the corrections to the wave vector due to ionization, and more specifically due to the free electron density induced in the medium. The wave vectors $k_{\Omega}, k_{\omega_{i}}$ are those describing propagation in the neutral me-
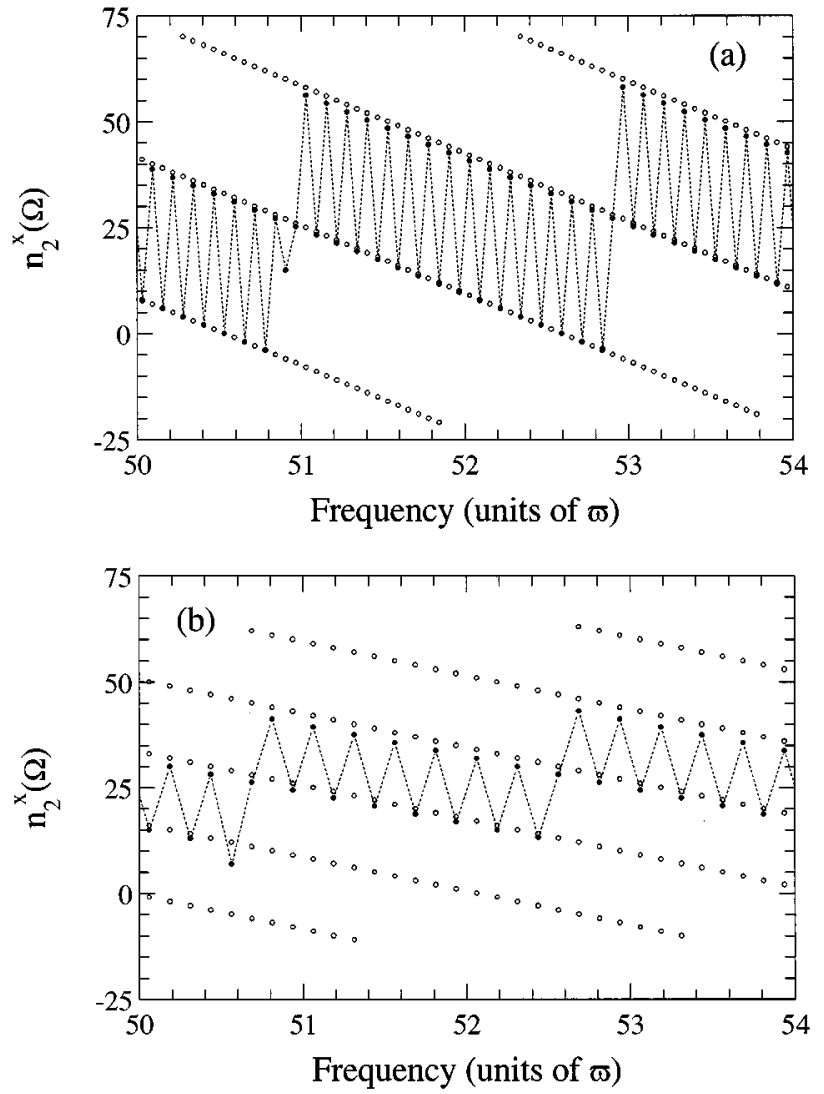

FIG. 8. Parameter $n_{2}^{x}(\Omega)$ (a) for $\Delta=\bar{\omega} / 32$ and (b) for $\Delta=\bar{\omega} / 16$, in solid circles. The open circles in both figures represent the solutions of the equation $\Omega=n_{1} \omega_{1}+n_{2} \omega_{2}$ for integers $n_{1}, n_{2}$, such that $\left|n_{1}\right|+\left|n_{2}\right|<\left(3 U_{p}+I_{p}\right) / \bar{\omega}$.

dium. Since the ionization rate $\Gamma$ depends on $\vec{E}_{\omega_{i}}\left(\vec{r}, t^{\prime}\right)$, Eqs. (12) have to be solved first, and their solution is used then to solve Eqs. (13).

In Fig. 7, we present the same result as in Fig. 4(d) but taking into account the depletion of neutral atoms and the dispersion effects due to the free electrons. We show two results, one obtained for a pulse width of 25 fs (solid line) and one for a pulse width of $150 \mathrm{fs}$ (dashed line). The comparison with the result of Fig. 4(b) (dot-dashed line) indicates that, in the regime of parameters used to investigate the generation of attosecond pulses, the effects due to depletion and ionization-induced dispersion do not play an important role.

\section{CONCLUSION}

In this paper we apply our theory of harmonic generation to study the generation of attosecond pulses in a laser field with a time-dependent degree of ellipticity, as proposed by Corkum and co-workers [4]. We show that, on the level of single-atom response, an efficient selection of a single attosecond pulse is hardly possible because of the existence of several electron trajectories leading to the harmonic emission. However, this effect can be controlled and significantly reduced in the process of propagation and buildup of the macroscopic harmonic signal in the medium. Due to the time filtering induced by propagation, and for a short enough laser 
pulse, selection of a single attosecond pulse becomes possible. The power in that single attosecond pulse is comparable to that of the pulses generated in the case of linear polarization. Finally, in the regime of parameters considered, dispersion and ionization effects do not much affect our results.

\section{ACKNOWLEDGMENTS}

$\mathrm{Ph} . \mathrm{A}$. is "collaborateur scientifique du Fonds National de la Recherche Scientifique de la Communauté Française de Belgique.' D.M. thanks the Soros Foundations, Open Society Fund Bosnia-Herzegovina, Research Support Scheme in Science. This work has been supported in part by the Swedish Natural Science Research Council.

\section{APPENDIX}

In this appendix, we describe how to obtain the dipole moment $\vec{x}_{\Omega}(\vec{r})$ for the field $\mathcal{E}_{0}(\vec{r})\left(\cos \left[\omega_{1} t\right.\right.$ $\left.+\phi(\vec{r})], \cos \left[\omega_{2} t+\phi(\vec{r})\right], 0\right)$ at the position $\vec{r}$. The phase $\phi(\vec{r})$ comes from focusing. In the following, we drop the $\vec{r}$ and denote $\vec{x}_{\Omega}(\vec{r})$ by $\vec{x}_{\Omega}(\phi)$. If the field had been monochromatic with frequency $\omega_{1}$, such a phase shift would have led to a time shift $\left(t_{0}=\phi / \omega_{1}\right)$ of the atomic response and the components $\vec{x}_{\Omega}(\phi)$ would have been simply related to the $\vec{x}_{\Omega}(0)$ by $\vec{x}_{\Omega}(\phi)=\vec{x}_{\Omega}(0) \exp \left(i \Omega \phi / \omega_{1}\right)$. In the present case, even if we assume the same focusing conditions for the two components of the field, we have $\omega_{1} t+\phi=\omega_{1}\left(t+t_{0}\right)$ and $\omega_{2} t+\phi=\omega_{2}\left(t+t_{0}\right)+\left(\omega_{1}-\omega_{2}\right) t_{0}$, i.e., a relative phase shift is acquired. This implies that the components $\vec{x}_{\Omega}(\phi)$ need to be calculated for all possible values of $\phi$.

Amazingly, in the regime of parameters of interest, our numerical calculations indicate that the expression for $\vec{x}_{\Omega}(\phi)$ takes an extremely simple form

$$
x_{\Omega}^{i}(\phi)=x_{\Omega}^{i}(0) \exp \left[i \phi_{\mathrm{eff}}^{i}(\phi, \Omega)\right],
$$

where $i$ refers to the $x$ or $y$ components and the effective phase is

$$
\phi_{\mathrm{eff}}^{i}(\phi, \Omega)=\frac{\Omega}{\omega_{1}} \phi+n_{2}^{i}(\Omega)\left(1-\frac{\omega_{2}}{\omega_{1}}\right) \phi
$$

In the above expression, $n_{2}^{i}(\Omega)$ is a parameter which depends only on the frequency of the considered Fourier component and which we determine numerically. The values of $n_{2}^{x}(\Omega)$ are plotted as solid circles in Fig. 8(a) for $\Delta=\bar{\omega} / 32$, and in Fig. 8(b) for $\Delta=\bar{\omega} / 16$. The open circles in both figures represent the values of $n_{2}$ that correspond to solutions of the equation $\Omega=n_{1} \omega_{1}+n_{2} \omega_{2}$ for integers $n_{1}, n_{2}$, such that $\left|n_{1}\right|+\left|n_{2}\right|<\left(3 U_{p}+I_{p}\right) / \bar{\omega}$. As we see, $n_{2}^{x}(\Omega)$ is typically very close to some of those solutions, so that in practice a simple analytical approximation to $n_{2}^{x}(\Omega)$ can be used. The results for $n_{2}^{y}(\Omega)$ are very similar.

The result of Eq. (A1) is important. It allows us to calculate $\vec{x}_{\Omega}(\phi)$ by knowing $\vec{x}_{\Omega}(0)$, and $n_{2}^{i}(\Omega)$. This reduces the amount of data to be generated and stored for the propagation problem enormously.
[1] Ph. Antoine, M. Gaarde, P. Salières, B. Carré, A. L'Huillier, and M. Lewenstein, in Proceedings of the VIIth International Conference on Multiphoton Processes, edited by P. Lambropoulos and H. Walther (Institute of Physics and Physical Society, Bristol, 1997).

[2] G. Farkas and C. Toth, Phys. Lett. A 168, 447 (1992); S. E. Harris, J. J. Macklin, and T. W. Hänsch, Opt. Commun. 100, 487 (1993)

[3] Ph. Antoine, A. L'Huillier, and M. Lewenstein, Phys. Rev. Lett. 77, 1234 (1996).

[4] P. B. Corkum, N. H. Burnett, and M. Y. Ivanov, Opt. Lett. 19, 1870 (1994); M. Yu. Ivanov, P. B. Corkum, T. Zuo, and A. Bandrauk, Phys. Rev. Lett. 74, 2933 (1995).

[5] K. J. Schafer and K. C. Kulander, Phys. Rev. Lett. 78, 638 (1997).

[6] I. P. Christov, M. M. Murnane, and H. C. Kapteyn, Phys. Rev. Lett. 78, 1251 (1997).

[7] This idea has also been discussed by P. Salières, Ph.D. thesis, Université Paris VI, 1995; also P. Salières, A. L'Huillier, Ph. Antoine, and M. Lewenstein, Adv. At., Mol., Opt. Phys. (to be published).

[8] K. S. Budil, P. Salières, Anne L'Huillier, T. Ditmire, and M. D. Perry, Phys. Rev. A 48, R3437 (1993).

[9] P. Dietrich, N. H. Burnett, M. Yu. Ivanov, and P. B. Corkum, Phys. Rev. A 50, R3585 (1994).

[10] C.-G. Wahlström, C. Altucci, Ph. Antoine, M. Bellini, M. B.
Gaarde, T. W. Hänsch, A. L'Huillier, C. Lyngå, I. Mercer, E. Mevel, and R. Zerne, in Proceedings of the VIIth International Conference on Multiphoton Processes, edited by P. Lambropoulos and H. Walther, IOP Conf. Ser. No. 154 (Institute of Physics and Physical Society, Bristol, 1997).

[11] P. Salières, A. L'Huillier, and M. Lewenstein, Phys. Rev. Lett. 74, 3776 (1995).

[12] For early work with $\mathrm{CO}_{2}$ lasers see R. L. Carman, D. W. Forslund, and J. M. Kindel, Phys. Rev. Lett. 46, 29 (1981).

[13] S. Kohlweyer, G. D. Tsakiris, C. G. Wahlström, C. Tillman, and I. Mercer, Opt. Commun. 117, 431 (1995).

[14] D. von der Linde, T. Engers, G. Jenke, P. Agostini, G. Grillon, E. Nibbering, A. Mysyrowicz, and A. Antonetti, Phys. Rev. A 52, R25 (1995).

[15] P. A. Norreys, M. Zepf, S. Moustaizis, A. P. Fews, J. Zhang, P. Lee, M. Bekarezos, C. N. Danzos, A. Dyson, P. Gibbon, P. Loukakos, D. Neely, F. N. Wajsh, J. S. Wark, and A. E. Angor, Phys. Rev. Lett. 76, 1832 (1996).

[16] K. Rzążewski, L. Plaja, L. Roso, and M. Lewenstein (unpublished).

[17] P. Gibbon, Phys. Rev. Lett. 76, 50 (1996).

[18] M. Lewenstein, Ph. Balcou, M. Yu. Ivanov, A. L'Huillier, and P. Corkum, Phys. Rev. A 49, 2117 (1994).

[19] W. Becker, A. Lohr, M. Kleber, and M. Lewenstein, Phys. Rev. A 56, 645 (1997).

[20] Ph. Antoine, A. L'Huillier, M. Lewenstein, P. Salières, and B. Carré, Phys. Rev. A 53, 1725 (1996). 
[21] It is worth stressing at this point that both the saddle-point approximation with respect to the momentum used in Eq. (1), and the use of $s$ states may introduce errors to our theory. In fact our approach reproduces quite accurately the form of the harmonic spectrum for noble gas atoms, but underestimates the numbers of photons emitted. Direct comparison of our results with the numerical solutions of the time-dependent Schrödinger equation indicates that our theory predicts typically photon numbers smaller by a factor 5-10 [see Ph. Antoine, B. Piraux, D. B. Milošević, and M. Gajda, Laser Phys. 7, 3 (1997). In D. B. Milošević and B. Piraux, Phys. Rev. A 54, 1522 (1996) it was checked that the next-order correction to the saddle-point approximation is of the order $10 \%$ and therefore it is not a critical one. We believe that the discrepancy between the strong-field approximation model and the numerical solutions of the time-dependent Schrödinger equation is mainly due to the Coulomb corrections to the dipole matrix element. Therefore we plan to improve the strong-field ap- proximation model by including these corrections $[\mathrm{Ph}$. Antoine, D. B. Milošević, and B. Piraux (private communication)].

[22] We also checked that we obtain very similar results by choosing a Gaussian filter function instead of this simple square window.

[23] M. Lewenstein, P. Salières, and A. L'Huillier, Phys. Rev. A 52, 4747 (1995).

[24] M. Protopapas, D. G. Lappas, C. H. Keitel, and P. L. Knight, Phys. Rev. A 53, R2933 (1996).

[25] J. B. Watson, A. Sanpera, K. Burnett, and P. L. Knight, Phys. Rev. A 55, 1224 (1997).

[26] C. Kan, C. E. Capjak, and R. Rankin, Phys. Rev. A 54, R1026 (1996).

[27] In practice, the expression (11) for the decay rate, as well as the formula (9) for the Fourier transform of the electronic polarization, contains a sum of contributions of all active electrons (i.e., two $s$ electrons for $\mathrm{He}$, six $p$ electrons for $\mathrm{Xe}, \mathrm{Ar}$, $\mathrm{Ne}$, etc.). 